\title{
BASIC STUDY ON THE DECAYED BEHAVIOR OF WASTE WOODY SAMPLES CAUSED BY THREE WHITE-ROT FUNGI
}

\author{
QINGYUE WANG, HIDEHARU TAKAHASHI, SAYURI KAWAMURA \& SHIN HATAKEYAMA \\ Graduate School of Science and Engineering, Saitama University, Japan
}

\begin{abstract}
In this study, we investigated the decayed behavior of waste woody samples caused by three kinds of white-rot fungi known as Coriolus consors, Coriolus hirsutus and Ischnoderma resinosum, which were selected to prepare and develop the decayed woody samples from original woody biomasses. After 8 weeks of decaying, the weights of all decayed woody samples had decreased. From the results of analysis of the major woody holocellulose and lignin, the same ratios of weight decrement in all decayed woody samples were found when compared with their original woody sample. It seemed that these components had degraded simultaneously. The crystallinities of woody samples had increased even though woody samples were decayed. It was thought that the amorphous parts of cellulose and hemicellulose were decomposed preferentially. We tried to use these decayed woody samples as samples for the liquefaction experiment. It is observed that the decayed woody samples may be liquefied to be the same as their original woody samples basing on the molecular weight distribution, chemical structure and other chemical parameters of the liquefied contents.

Keywords: characterization, decayed waste woody samples, white-rot fungus.
\end{abstract}

\section{INTRODUCTION}

The consumption and depletion of fossil fuel is an important social issue. Considering the increasing trend of population and the rising use of resources, the development of new alternative resources such as fossil fuel is necessary. As a renewable resource, the use of biomass has been developed in considering a clean environment and renewable circular resources. Biomass is separated into three types: waste biomass [1], [2], unused biomass, and crop resources. For considering woody material, thinned woody biomass and industrial waste woody biomass have lower utility values and cannot be utilized. Furthermore, such woody biomasses often decay due to degradation by wood-rot fungus, making the materials less suitable for use. But those unused woody biomasses can have high added values when liquefied. Wood liquefaction is one of the most effective technologies for biomass utilization [3]. When a woody powder is mixed with an organic solvent in the presence of an acid catalyst and heated, a black viscous liquid material is formed. This material is called liquefied wood and can form resin by adding chemical reagents [4]-[6]. Actually, although there are some samples that can provide an alternative to traditional resin, most of these are used as organic solvents above twice as much as woody biomasses. One of the merits of liquefaction is saving oil resources; therefore, a higher rate of biomass is preferred. One of the reasons for this is the effect of condensation [7]-[9]; pre-treatment is used to inhibit it [10]-[12]. In this way, liquefaction is the decomposition of the main component using a solvent and catalyst, and decay is much the same process, but by wood-rot fungus. This means that decay can be considered as a pre-treatment process. The woody samples that had decayed by brown-rot fungus - one classification in three large groups - were liquefied better than their original woody sample because of a relatively large amount of lignin following the decomposition of cellulose and the destruction of crystallinities of cellulose [13]. Additionally, in previous work the waste woody samples that had decayed by white-rot fungus had separated from their non-decayed parts and liquefied, which indicated that the decayed wood can liquefy in 
the same way as the non-decayed wood [14]. Since there is less information about white-rot fungus, in this study, for the preparation of liquefying the decayed wood, the woody samples were decayed by white-rot fungus, and the weight loss and morphologic changes were measured.

\section{MATERIAL AND METHODS}

\subsection{Preparation of decayed woody samples}

Three species of white rot fungus, Coriolus consors, Coriolus hirsutus and Ischnoderma resinosum (C. consors, C. hirsutus and I. resinosum, from the Institute of Wood Technology, Akita Prefectural University, Japan) were prepared for wood-decay examination and cultured by using potato dextrose agars (PDA). The beech wood (Fagus crenata) was crushed and classified to $<250 \mu \mathrm{m}$ through a sieve. Then, $20 \mathrm{~g}$ of this woody powder and $40 \mathrm{~mL}$ of pure water were set in a culturing bottle. Then the cap of this bottle was closed loosely and autoclaved at $121^{\circ} \mathrm{C}$ for 15 minutes. After autoclaving, four pieces of hyphae of the cultivated white-rot fungus were put into the culturing bottle together with the PDA. The culturing bottles were used for culturing the white-rot fungus (C. consors, C. hirsutus and I. resinosum) and the control samples were only wood without any white-rot fungus. Each sample had four replicates. These culturing bottles were left to rest in a plant growth chamber (Biotron LPH$350 \mathrm{SP}$, Nippon Medical and Chemical Instruments Co. Ltd., Japan) configured at $25^{\circ} \mathrm{C}$ and $90 \%$ relative humidity for 8 weeks. After decay examination, the grown hyphae were removed carefully. The woody powder samples were rinsed with pure water and dried at $105^{\circ} \mathrm{C}$ overnight, then their weights were measured.

\subsection{Analysis of components of decayed woody samples}

First, to analyze the holocellulose (cellulose and hemicellulose) and lignin of the woody samples, the samples were defatted using Soxhlet extraction. A total of $50 \mathrm{~mL}$ of ethanol and $100 \mathrm{~mL}$ of benzene were used for making the defatted woody samples.

To analyze the content ratio of holocellulose, we conducted examinations using the following method: $2.5 \mathrm{~g}$ of the defatted woody samples were put in a $300 \mathrm{~mL}$ flask with 150 $\mathrm{mL}$ of pure water, $1.0 \mathrm{~g}$ of sodium chlorite and $0.20 \mathrm{~mL}$ of acetic acid, which were heated at $75^{\circ} \mathrm{C}$ in a water bath; with occasional stirring, $1.0 \mathrm{~g}$ of sodium chlorite and $0.2 \mathrm{~mL}$ of acetic acid were added hour after hour, three times; then these contents were filtered used a glass filter (1GP100, AS ONE Co. Ltd., Japan) and rinsed with pure water and acetone. The residual solid was holocellulose.

To analyze the content ratio of lignin, we examined by the Klason lignin method. Some $1.00 \mathrm{~g}$ of the defatted woody samples and $15 \mathrm{~mL}$ of $72 \%$ sulfuric acid were mixed with $50 \mathrm{~mL}$ in a beaker for 4 hours, with occasional stirring. These contents were poured into a $1 \mathrm{~L}$ Erlenmeyer flask which was filled with $560 \mathrm{~mL}$ of extra-pure water, little by little. This flask was fitted with a condenser and heated at $110^{\circ} \mathrm{C}$ in an oil bath for 4 hours. Then, these contents were filtered using a glass filter (1GP16, AS ONE Co. Ltd., Japan) and rinsed with hot water and pure water. The residual solid was lignin.

\subsection{Measurement of crystallinity of woody samples using X-ray diffraction}

Cellulose is known to have a crystal-like structure. It is possible to decrease the crystallinity of cellulose with the degradation by wood-rot fungus. Therefore, using X-ray diffraction (XRD) (Rint UltimaIII, Rigaku Corporation, Japan), the crystallinity results of the woody 
samples were measured. The holocellulose of woody samples was used for the XRD sample. Additionally, a baseline was pulled between the intensity values of $10^{\circ}$ and $30^{\circ}$ of the diffraction angle, and the crystallinity was calculated as shown in the following eqn (1), as the area above baseline was the crystalline region and the area under the baseline was the amorphous region $10^{\circ}$ and $30^{\circ}$ of diffraction angle.

$$
\text { Crystallinity }(\%)=\frac{S_{c}}{S_{c}-S_{a}} \times 100
$$

Sc: the area of crystal region;

$S a$ : the area of amorphous region.

2.4 Measurement of chemical structure of woody samples using a Fourier transform infrared spectroscopy (FT-IR)

By measuring Fourier transform infrared spectroscopy (FT-IR JASCO Co. Ltd., Japan), it is possible to know which functional group changed because of white-rot fungus. The woody samples were mixed with $\mathrm{KBr}$ powder $(5: 95, \mathrm{w} / \mathrm{w})$. A small amount of the mixed powder sample was stuffed in a special pellet and then pressed. The pellet was set in FT-IR and the functional groups of woody samples were measured.

\section{RESULTS AND DISCUSSION}

\subsection{Decay examination}

\subsubsection{Decay of woody samples}

Photos of the sample of I. resinosum before and after decay are shown in Fig. 1. In the initial step of the examination, the hypha existed only on the PDA fragments. Afterwards, in the final step of the examination, the hypha entirely outspread in the woody powder and the woody powder turned slightly white. The sample of $C$. hirsutus also showed similar results. Although the hypha of the sample of $C$. consors did not outspread on the surface of the woody powder, the hyphae reached near the bottom of the bottle.

\subsubsection{Decrease ration of decay woody}

Fig. 2 shows the rate of weight change between the before and after examination by measuring the cleaning and drying weight of powder after examination and removing hypha. Weights decreased in the control treatment, which might be caused by the inadequate drying before the examination, where the total samples would be in consideration of this decreasing trend. The weight of all samples of white-rot fungus decreased and the difference is nearly $10 \%$. It shows the hypha of $C$. consors also reached the interior of the woody powder and the samples of $C$. consors, C. hirsutus and I. resinosum were rotten.

\subsection{Decay examination}

\subsubsection{Decay of woody samples}

Fig. 3 shows the results of component analysis. Although holocellulose seemed to be similar with the sample of the control (Control), lignin had decreased a little. The absolute weight including the weight decreased by the decay examination have to considered because the result of this investigation concerns the content of a component. Fig. 4 shows the results reflected as the absolute weight. However, this way, both holocellulose and ligunin decreased in the samples of C. consors, C. hirsutus and I. resinosum because of the decrease of the 
weight when they were compared with the Control. The reason why the rate of the component did not change, in spite of the decrease of weight, is that white-rot fungus rotted each component at the same time. Kirk and Highley [15] suggested that the main components of wood are decomposed at the same time by white-rot fungus. Similarly, each component can be decomposed at the same time, and weight was obviously decreased. The lignin components were decreased because lignin was amorphous or low crystalline. Therefore, the decomposition rate was relatively fast.
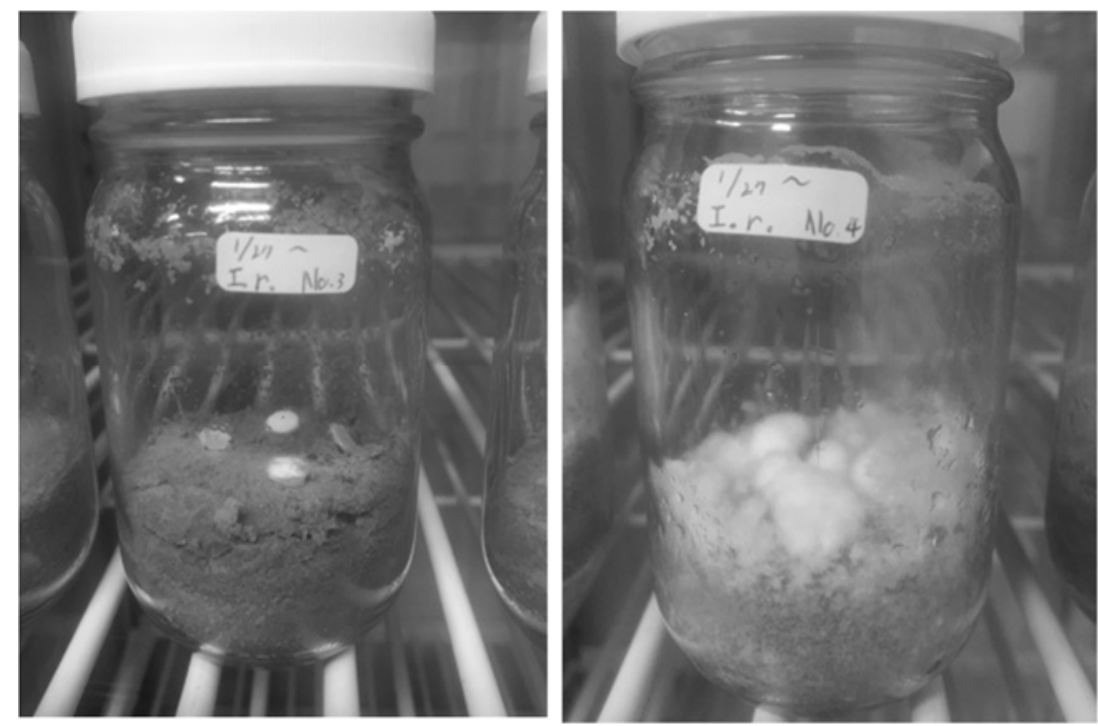

Figure 1: Photos of before (left) and after (right) decay examination; decayed by Ischnoderma resinosum.

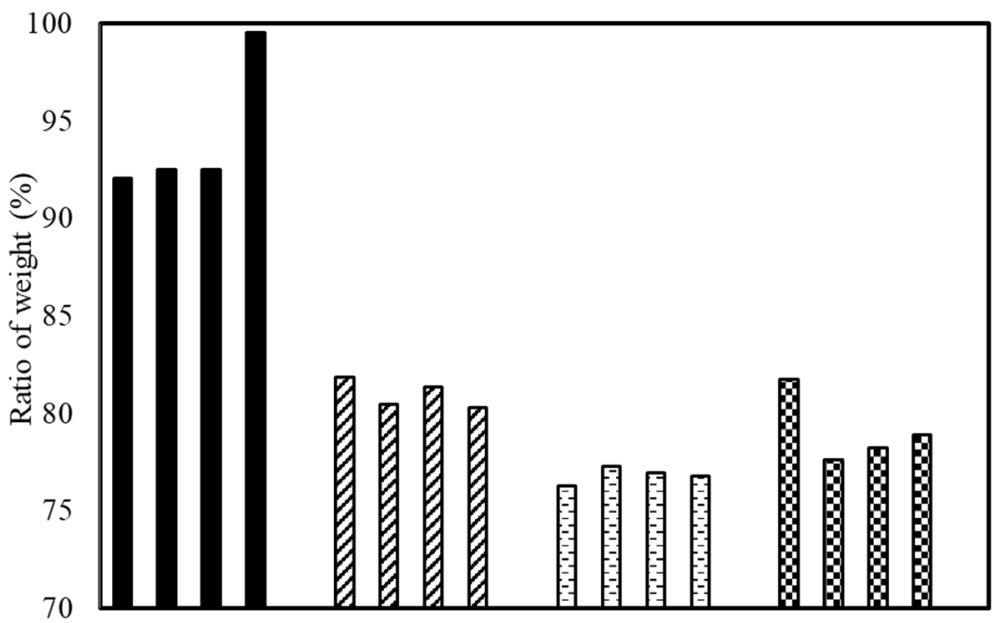

Figure 2: The ratios of the weight of the woody samples after decay examination (original control woody sample, C. consors, C. hirsutus and I. resinosum). 


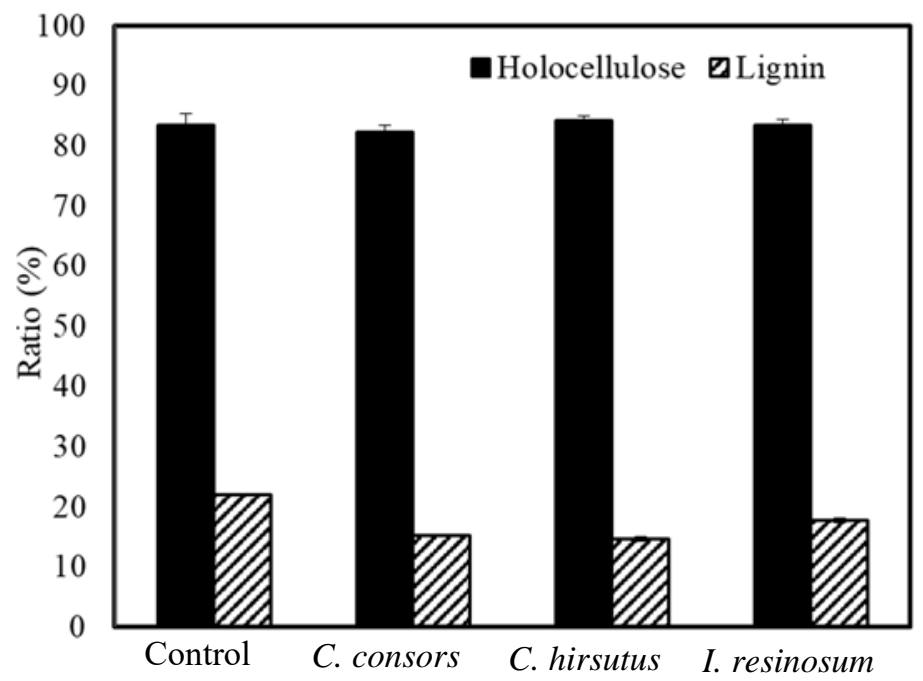

Figure 3: The ratios of wood samples (original control woody sample, C. consors, C. hirsutus and I. resinosum).

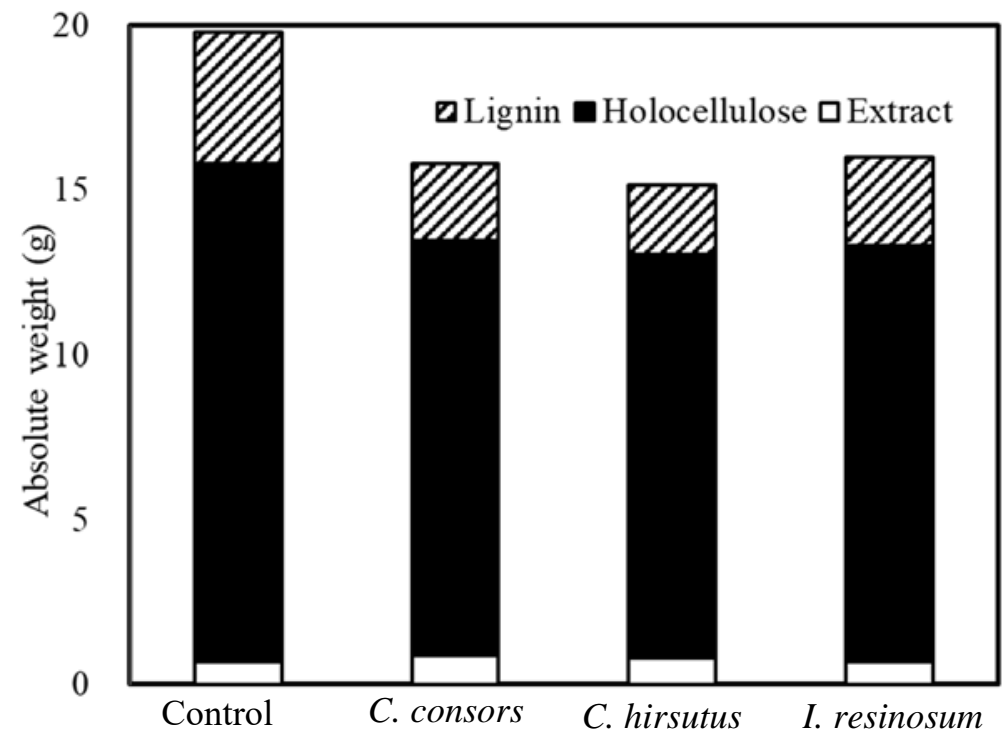

Figure 4: The absolute weight of the woody samples (original control woody sample, C. consors, C. hirsutus and I. resinosum).

\subsubsection{Crystallinity}

Fig. 5 shows the result of the XRD spectra of the woody samples. The strength of peak of decayed wood seems to be increased rather than decreased by calculating from the peak XRD used. Table 1 shows the crystallinity calculated from the peak. 


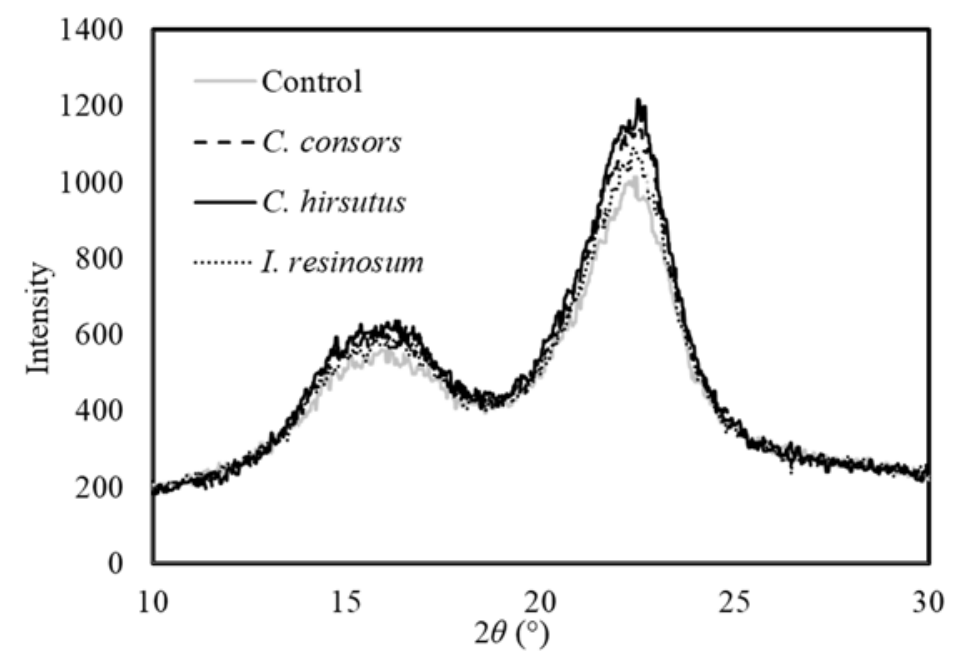

Figure 5: The XRD spectra of the woody samples (original control woody sample, $C$. consors, C. hirsutus and I. resinosum).

Table 1: The crystallinity of the decayed woody and original control woody samples.

\begin{tabular}{|l|l|}
\hline Woody sample & Crystallinity (\%) \\
\hline Control & 51.3 \\
\hline C. consors & 56.2 \\
\hline C. hirsutus & 55.3 \\
\hline I. resinosum & 47.0 \\
\hline
\end{tabular}

The crystallinity of $C$. consors and $C$. hirsutus is better than that of the original control woody sample. The crystallinity increased, against the expectation that it would decrease by the breaking of the crystal structure of cellulose as a result of a wood-rotting fungus such as I. resinosum. This occurred because non-crystalline cellulose has also decayed as a priority. Howell et al. [16] show crystallinity increased at the initial step in a term of decay using brown-decay fungus when compared with that of another type of decay. Hastrup et al. [17] showed that in the case of white-rot fungus. there was also an increase in crystallinity and they suggested that occurred because they decomposed non-crystalline as a priority. It is suggested that crystallinity increases because of the shorter term of decay in this study.

\subsubsection{Chemical structure of the decayed woody samples}

Fig. 6 shows the results of the chemical structure of the decayed woody samples measured using FT-IR. All decayed woody samples show the same peak when compared with the Control; the reason for this slight deference was considered to be the decomposition of wood composition, which occurred at the same time. Pandey and Pitman [18] analyzed decayed wood by brown-decay fungus and white-decay fungus using FT-IR and revealed that the decayed wood changed when exposed to brown-decay fungus, but did not change when it was exposed to white-decay fungus - the reason for this is the same time for decay was used for the white-decay fungus. No changes were observed in this study for the reason. 


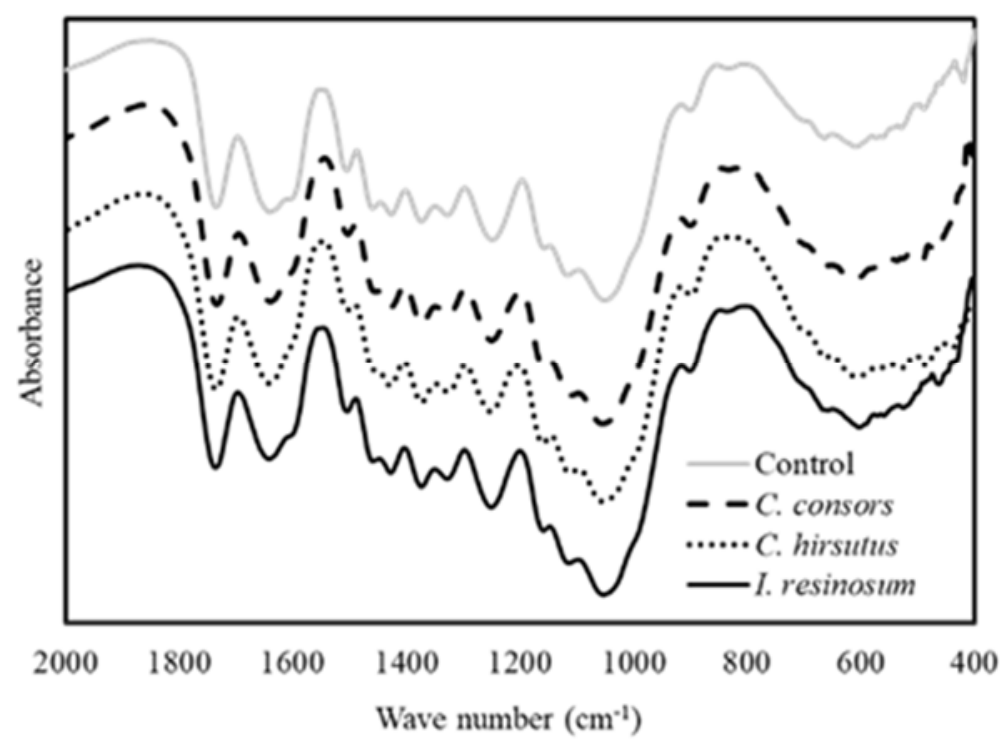

Figure 6: The FT-IR spectra of the decayed woody samples (original Control woody sample, C. consors, C. hirsutus and I. resinosum).

\subsection{Examination of large particle sizes of the woody samples}

\subsubsection{Decay examination}

The photos of the before and after decay examination (the sample of $C$. hirsutus) are shown in Fig. 7. The fungal thread grew and became larger than the samples examined for 2 months which could be found in the woody powder. Fig. 8 shows the rate of weight change by taking measurements after removing the fungal thread, cleaning with pure water and drying for one night. There is almost no change in the Control sample because it was adequately dried. We found three differences in the conditions of the large and small particle sizes of the woody samples: the size is larger, the decay term is one-month longer, liquid culture medium is used for kindling fungus, and the result is a decrease of more than $20 \%$ in this examination, in contrast to the net $10 \%$ decrease. By using these wood-powder samples, the study of more decayed wood can be carried out.

\subsubsection{Analysis of components}

Fig. 9 shows the results of our analysis of the components. In spite of large sizes and high rations of decay, the results of the analysis of the components found no significant difference between the decayed wood and control wood samples. This result suggests that the decaying of each component is carried in the same time as the above examination. The results of holocellulose in decayed wood are a little higher and lignin is a little lower than those of the Control because of the little difference between cellulose having crystallinity and lignin not having crystallinity. Fig. 10 shows the absolute weight. Considering weight loss, the main components of the wood, holocellulose and lignin, decrease and this means these components are converted by liquefaction and disappear. This has to be considered for other examinations of pre-processing. 


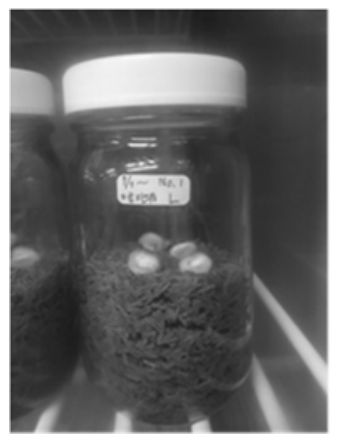

(a)

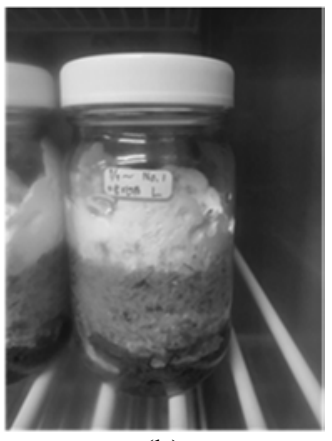

(b)

Figure 7: The decay examination of the woody samples of large particle size. (a) On the first day; (b) On the last day.

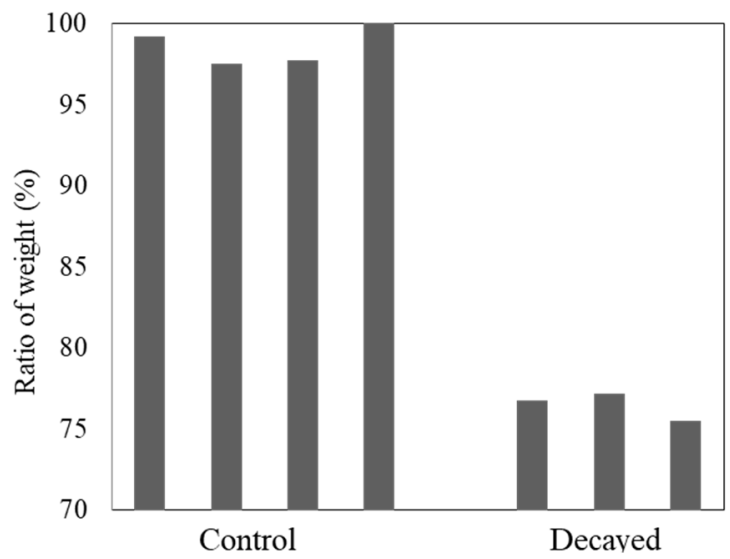

Figure 8: The ratios of weight of the woody samples of large particle sizes after decay.

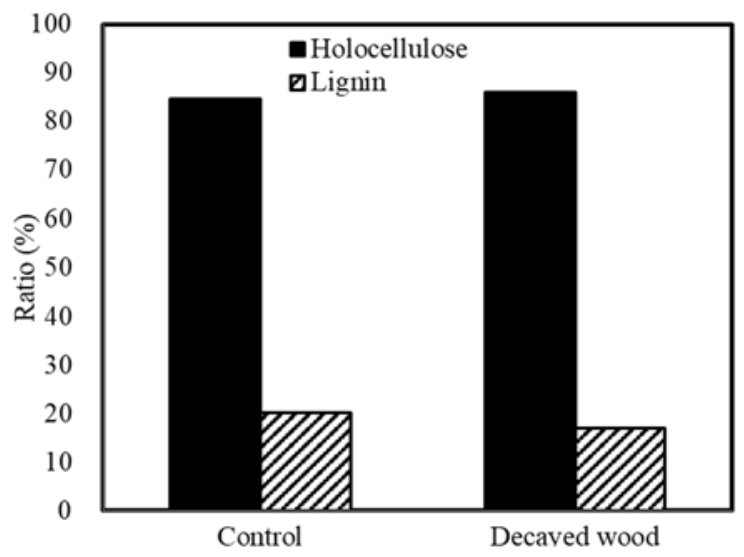

Figure 9: The ratios of wood components of large particle sizes. 


\subsubsection{Crystallinity}

Fig. 11 shows the XRD measuring results and Table 2 shows the calculated results of crystallinity. In this examination, the peak of the decayed wood is lower than the original Control woody sample in contrast to the small size. The crystallinity of decayed wood was lower than the original Control woody sample, as shown in the Table 2, as the peak shows. It was thought that, because of sufficient long-term decay, it would be easier to grow larger sizes of particle size and with the addition of liquid culture medium, the crystallinity would decrease beyond term of increases, relatively.

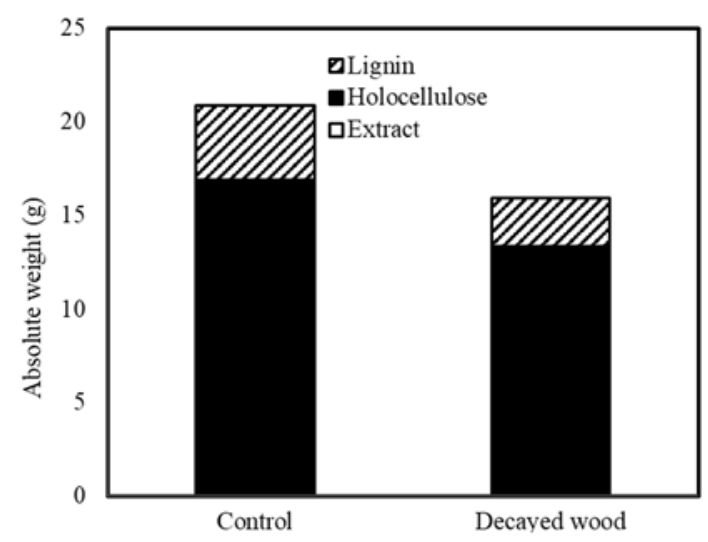

Figure 10: The absolute weight of the woody samples of large particle sizes.

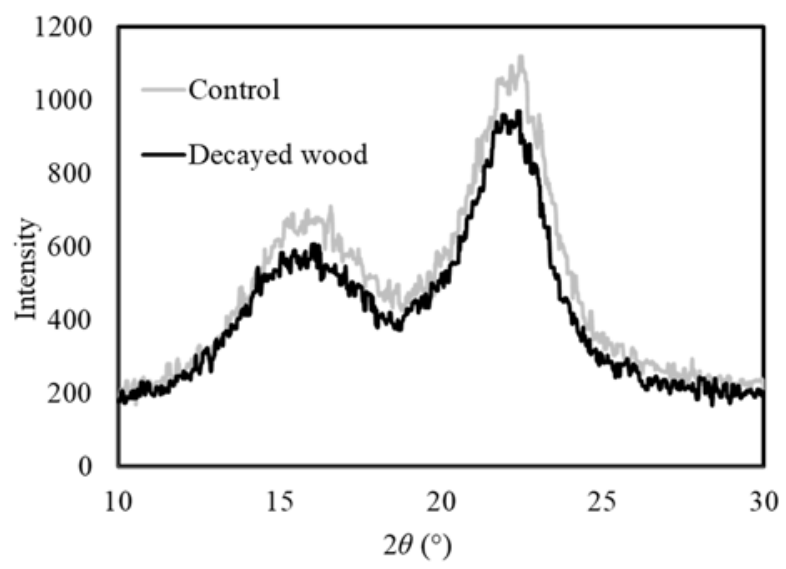

Figure 11: The XRD spectra of the woody samples of large particle sizes.

Table 2: The crystallinity of the woody samples of large particle sizes.

\begin{tabular}{|l|l|}
\hline Woody sample & Crystallinity (\%) \\
\hline Control & 63.8 \\
\hline Decayed wood & 57.6 \\
\hline
\end{tabular}




\section{CONCLUSION}

Three kinds of white-decay fungi (Coriolus consors, Coriolus hirsutus and Ischnoderma resinosum) were used for this study. The weight reduction of all decayed wood samples was determined after eight weeks of decay examination. The content rate of holocellulose and lignin, the main components of wood, were almost the same as original Control woody sample because decomposition and absorption occurred simultaneously by white-decay fungus. Compared with the crystallinity, the group with the fungus showed higher levels than the original Control woody sample. This result might be caused by the amorphous part. These results show that decayed wood by white-decay fungus may be liquefied in the same way as the original wood.

\section{ACKNOWLEDGEMENT}

Some of this work was supported by the Special Funds for Basic Research (B) (no. 15H05119, FY2015 FY2017) of Grant-in-Aid Scientific Research of the Japanese Ministry of Education, Culture, Sports, Science and Technology (MEXT), Japan.

\section{REFERENCES}

[1] Wang, Q., Qiao, Q., Chen Q. \& Sugiyama, K., Process analysis of the waste bamboo by using polyethylene glycol solvent liquefaction. International Journal of Sustainable Development and Planning, 9, pp. 647-657, 2014.

[2] Wang, Q. et al., Process analysis of waste bamboo materials using solvent liquefaction. WIT Transactions on Ecology and the Environment, 176, pp. 267-277, 2013.

[3] Shiraishi, N., Onodera, S., Ohtani, M. \& Masumoto, T., Dissolution of etherified or esterified wood into polyhydric alcohols or bisphenol A and their application in preparing wooden polymeric materials. Mokuzai Gakkaishi, 31, pp. 418-420, 1985.

[4] Yan, Y., Pang, H., Yang, X., Zhang, R. \& Liao, B., Preparation and characterization of water-blown polyurethane foams from liquefied cornstalk polyol. Applied Polymer Science, 110, pp. 1099-1111, 2008.

[5] Lee, S.H., Teramoto, Y. \& Shiraishi, N., Resol-type phenolic resin from liquefied phenolated wood and its application to phenolic foam. Journal of Applied Polymer Science, 84, p. 468, 2001.

[6] Wang, Q. et al., Liquefaction processes and characterization of liquefied products from waste woody materials in different acidic catalysts. The Sustainable World, Ecology and the Environment, 142, pp. 343-354, 2010.

[7] Wang, Q. et al., Investigation of condensation reaction during phenol liquefaction of waste woody materials. International Journal of Sustainable Development and Planning, 9, pp. 658-668, 2014.

[8] Wang, Q. et al., Suppression method of the condensation reaction during phenol liquefaction of woody material. WIT Transactions on Ecology and the Environment, 176, pp. 1743-3541, 2013.

[9] Wang, Q., Effect of the phenol concentrations on the condensation reaction during the liquefaction of waste woody materials with phenol. Waste Management and the Environment VI, Ecology and The Environment, 163, pp. 355-366, 2012.

[10] Wang, Q., Chen, Q., Mitsumura, N. \& Niida, H., Improved cellulose by ionic liquidwater mixture with solid acid catalysis and its application in polyethylene glycol liquefaction. Materials Sciences and Applications, 5, pp. 183-192, 2014.

[11] Chen, Q. \& Wang, Q., Application of ionic liquid and water mixture in cellulose solvent liquefaction as pretreatment step. Biomass Chemical Engineering, 48, pp. 1-8, 2014. 
[12] Wang, Q., Chen, Q., Mitsumura, N. \& Animesh, S., Behavior of cellulose liquefaction after pretreatment using ionic liquids with-water mixtures. Journal of Applied Polymer Science, 131, pp. 1-8, 2013.

[13] Li, G.Y., Hse, C.Y. \& Qin, T.F., Preparation and characterization of novolak phenol formaldehyde resin from liquefied brown-rotted wood. Journal of Applied Polymer Science, 125, pp. 3142-3147, 2012.

[14] Takahashi, H., Takahashi, Y., Kurokawa, H., Sekiguchi, K. \& Sugiyama, K., Characterization of liquefied waste bamboo and white-rotted wood. Energy and Sustainability, 6, pp. 63-73, 2015.

[15] Kirk, T.K. \& Highley, T.L., Quantitative changes in structural components of conifer woods during decay by white- and brown-rot fungi. Phytopathology, 63, pp. 13381342, 1973.

[16] Howell, C., Christine, A., Hastrup, S., Goodell, B. \& Jellison, J., Temporal changes in wood crystalline cellulose during degradation by brown rot fungi. International Biodeterioration \& Biodegradation, 63, pp. 414-419, 2009.

[17] Hastrup, A.C.S., Howell, C., Larsen, F.H., Sathitsuksanoh, N., Goodell, B. \& Jellison, J., Differences in crystalline cellulose modification due to degradation by brown and white rot fungi. Fungal Biology, 116, pp. 1052-1063, 2012.

[18] Pandey, K.K. \& Pitman, A.J., FTIR studies of the changes in wood chemistry following decay by brown-rot and white-rot fungi. International Biodeterioration \& Biodegradation, 52, pp. 151-160, 2003. 